\title{
Presencia, actividad, visibilidad e interdisciplinariedad del profesorado universitario de Documentación en los medios sociales: una perspectiva de género
}

\author{
Michela Montesi*, Isabel Villaseñor Rodríguez*, Fernando Bittencourt dos Santos** \\ * Facultad de Ciencias de la Documentación, Universidad Complutense de Madrid \\ Correo-e: mmontesi@ucm.es | ORCID iD: https://orcid.org/0000-0002-5509-2075 \\ Correo-e: isavilla@ucm.es | ORCID iD: https://orcid.org/0000-0002-6000-7824 \\ ** Universidade Federal de Sergipe, Brasil \\ Correo-e: fernandoubatuba@hotmail.com | ORCID iD: https://orcid.org/0000-0003-1305-4262
}

Recibido: 11-01-2019; 2a versión: 27-02-2019; Aceptado: 04-03-2019.

\begin{abstract}
Cómo citar este artículo/Citation: Montesi, M.; Villaseñor Rodríguez, I.; dos Santos, F. B. (2019). Presencia, actividad, visibilidad e interdisciplinariedad del profesorado universitario de Documentación en los medios sociales: una perspectiva de género. Revista Española de Documentación Científica, 42 (4), e246. https://doi.org/10.3989/redc.2019.4.1640
\end{abstract}

Resumen: Se estudia la presencia, actividad, visibilidad e interdisciplinaridad de 349 profesoras y profesores de Documentación en los medios sociales, para comprobar si existen diferencias entre los dos conjuntos. Las plataformas estudiadas incluyen ResearchGate (RG), Google Scholar Citations (GSC), y Twitter, y la población analizada corresponde al profesorado de 13 universidades españolas que ofertan formación en Biblioteconomía y Documentación. Los datos se recogieron entre abril y junio de 2018. Para las cuatro dimensiones de estudio se analizaron diferentes variables, incluyendo, entre otros, el número de documentos subidos a RG y el número de tweets, respuestas y retweets en Twitter para la actividad, y el porcentaje de documentos en acceso abierto y de documentos diferentes a los géneros tradicionales en RG y el número de seguidores y seguidoras por perfil en Twitter para la visibilidad. Los resultados apuntan a diferencias entre los dos colectivos, especialmente acentuadas en términos de visibilidad.

Palabras clave: medios sociales académicos; sesgo de género; actividad investigadora; métricas alternativas; Biblioteconomía y Documentación.

Presence, activity, visibility and interdisciplinarity of Library and Information Science faculty on social media: a gender perspective

\begin{abstract}
The paper analyzes the presence, activity, visibility and interdisciplinarity of 349 Library and Information Science (LIS) faculty on social media, in order to find possible gender differences. Data were obtained between April and June 2018 from ResearchGate (RG), Google Scholar Citations (GSC), and Twitter, and the population studied corresponded to LIS faculty affiliated to 13 Spanish universities. Different variables were analyzed for the four dimensions, including, among others, the number of documents uploaded to RG and the number of tweets, replies and retweets on Twitter for the activity, along with the percentage of open access documents and of documents differing from traditional genders on RG and the number of followers and followees on Twitter for visibility. Results point out differences between the two groups, especially in terms of visibility.
\end{abstract}

Keywords: academic social media; gender bias; research activity; alternative metrics; Library and Information Science.

Copyright: () 2019 CSIC. Este es un artículo de acceso abierto distribuido bajo los términos de la licencia de uso y distribución Creative Commons Reconocimiento 4.0 Internacional (CC BY 4.0). 


\section{INTRODUCCIÓN}

La evaluación de la actividad investigadora contempla métricas a nivel individual cada vez más articuladas en un amplio abanico de indicadores, entre otros, muchos procedentes de los medios sociales (Orduña-Malea y otros, 2016). Desde una perspectiva de género, cabe interrogarse sobre el comportamiento de los nuevos indicadores y sobre la posibilidad de diferencias entre hombres y mujeres parecidas a las que se han detectado en la investigación previa para los indicadores más tradicionales. Los sesgos de los indicadores bibliométricos tradicionales se han resumido bajo lemas como el del "puzle de la productividad", para aludir a las diferencias cuantitativas en la producción científica entre hombres y mujeres (Leahey, 2006; Larivière y otros, 2011; Larivière y otros, 2013), o "el efecto Matilda", en referencia al impacto más destacado que la producción científica realizada por hombres suele tener en términos de citas recibidas (Knobloch-Westerwick y Glynn, 2013), por lo menos en ciertos ámbitos de conocimiento. Sin embargo, el sesgo de género parece afectar no solo a los indicadores bibliométricos, sino a procesos y actividades de la comunicación científica, como, por ejemplo, la presencia en los comités editoriales de revistas científicas (Mauleón y otros 2013) o los procesos de revisión por pares. En el trabajo de Helmer y otros (2017), la composición del personal de revisión de 142 revistas del portal Frontiers Journals en el periodo 2007-2015 presentaba una infrarrepresentación de mujeres, por un lado, y cierta "homofilia" o inclinación de quien ejerce el rol editorial a seleccionar revisores de su mismo sexo, por otro. Los patrones de citación también responden al criterio de la homofilia, por lo menos en ciertas áreas como las ciencias políticas o la comunicación, en las cuales los autores tienden a citar más a otros autores que autoras (Knobloch-Westerwick y Glynn, 2013; Maliniak y otros, 2013; Mitchell y otros, 2013).

Por otro lado, sabemos menos acerca de los posibles sesgos de género de los indicadores derivados de los medios sociales o, en general, de las diferencias de género relacionadas con el uso de los medios sociales para fines académicos. Paul-Hus y otros (2015) estudian el impacto de la actividad investigadora desde una perspectiva de género, comparando eventos en Twitter, blogs y menciones en las noticias para dos conjuntos de artículos, cuyo primer autor fuera respectivamente mujer u hombre. Encuentran que, en los medios sociales, los indicadores altmétricos parecen presentar un sesgo de género menor que las métricas tradicionales siendo más justos para las publicaciones del personal de investigación femenino, aunque con diferencias entre disciplinas. En cuanto al uso de los medios sociales con fines académicos, hace casi una década, Procter y otros (2010) analizaban la adopción de las herramientas de la web social en la comunidad académica del Reino Unido, encontrando que las mayores tasas de adopción estaban relacionadas con una mayor edad, una posición más afianzada, y el género, representando las mujeres la mayoría de las personas inactivas. En el trabajo de Tsou y otros (2016) los perfiles correspondientes a varones, mayores de 35 años y de raza caucásica, también dominaban en una muestra de más de 10.000 perfiles seleccionados de Google Scholar, Microsoft Academic Search y Mendeley, aunque con diferencias entre las tres plataformas, siendo GSC la que menos mujeres tenía y Mendeley la que más. Sugimoto y otros (2017) revisan la literatura disponible sobre el uso de los medios sociales con fines académicos, apuntando, entre otras cosas, a algunas diferencias de género en el uso de las plataformas sociales. A pesar de la escasez de investigaciones sobre un posible uso diferencial de las herramientas de la web social, Sugimoto y otros (2017) concluyen que, en ciertos ámbitos, como la publicación de blogs con contenidos científico-técnicos, existe un predominio masculino, mientras que en cuanto al uso de plataformas de microblogging los resultados no son definitivos sobre una posible brecha de género. Estudios de más amplio alcance con la población usuaria de algunas plataformas como Twitter muestran unas disparidades de género en términos de visibilidad más que de presencia. Messias y otros (2017), por ejemplo, encuentran que, en una muestra aleatoria de usuarios americanos de Twitter, aunque la población femenina representaba algo más de la mitad (52.69\%), los perfiles con más visibilidad, en términos de seguidores y de menciones en listas de usuarios, correspondían a varones de raza caucásica, identificando también un sesgo en cuanto a la raza y confirmando estudios previos como (Nilizadeh y otros, 2016). Dependiendo de la plataforma y de la muestra estudiada, la población femenina resulta infrarrepresentada en términos de presencia también. Centrándose únicamente en el personal docente e investigador de la Universidad de Bergen, Mikki y otros (2015) encuentran una infrarrepresentación del personal femenino en cinco redes sociales y plataformas académicas incluyendo ORCID y ResearcherID, especialmente en GSC y ResearcherID. Ortega (2017) encuentra diferencias en la presencia del personal de investigación del Consejo Superior de Investigaciones Científicas (CSIC) en tres plataformas sociales de corte académico, Academia. 
edu, ResearchGate (RG) y Google Scholar Citations (GSC), tanto en términos de género como de disciplinas, aunque constata cierta coherencia con la propia composición del mismo personal de investigación de la institución. Solo en el caso de Google Scholar Citations la población femenina estaría infrarrepresentada respecto a su participación real en la plantilla del CSIC. Para el colectivo de investigación en salud peruano, Jhonnel Alarco y otros (2016) encuentran que, de 200 perfiles de alto impacto analizados en Google Académico, solo el $15 \%$ corresponde a mujeres. Thelwall y Kousha (2014) valoran las diferencias de género en el análisis de los perfiles de Filosofía en el sitio Academia.edu, encontrando que los perfiles correspondientes a mujeres tendían a atraer un número menor de visitas. Al contrario, los perfiles femeninos en Derecho e Informática tendían a atraer un mayor número de visitas respecto a los compañeros.

También existen diferencias de género en ciertos patrones de la actividad investigadora que repercuten en los indicadores de impacto, como, por ejemplo, la colaboración o la especialización y la interdisciplinariedad. Las necesidades de la conciliación familiar pueden explicar la propensión del personal de investigación femenino a colaborar local más que internacionalmente, limitando el alcance y el impacto de los resultados obtenidos (Larivière y otros, 2011; Abramo y otros, 2013). Por otro lado, los equipos que cuentan con la colaboración de una proporción mayor de mujeres, incluso cuando publican en revistas de alto impacto, se ven afectados en términos de menores niveles de citación (Beaudry y Larivière, 2016). Finalmente, la investigación en Nanotecnología con vocación solidaria contempla patrones de colaboración diferentes dependiendo de si el primer autor es hombre o mujer, por lo menos en el contexto de Canadá (Ghiasi y otros, 2018). En el estudio de Ghiasi y otros (2018), los equipos encabezados por mujeres tienden a ser más equilibrados en término de sexos, mientras que los encabezados por hombres tenderían a ser exclusivamente masculinos. A la misma conclusión llegan Araújo y otros (2017), estudiando los patrones de colaboración de 270.000 científicas y científicos registrados en la Plataforma Lattes para diferentes disciplinas.

La especialización y, sobre todo, la interdisciplinariedad, están relacionadas con la colaboración, pues esta supone el encuentro de culturas epistemológicas diferentes para la resolución de problemas del mundo real. Ya Leahey (2006) explicaba las diferencias en productividad entre hombres y mujeres por una diferente especialización, por lo menos en Lingüística y Sociología. Los hombres tendían a centrarse en un solo dominio de conocimiento, aprovechando su base de conocimiento en la trayectoria de publicación y construyendo redes de contactos, mientras que las mujeres consideraban que la diversificación de los temas de investigación ampliaba sus identidades profesionales. Potthoff y Zimmermann (2017) achacan la homofilia en los patrones de citación del área de la comunicación a las diferentes especializaciones temáticas de mujeres y hombres. Por otro lado, el estudio de Van Rijnsoever y Hessels (2011) con el personal docente e investigador de la Universidad de Utrecht en los Países Bajos, revelaba que la colaboración interdisciplinaria era más común entre las mujeres, en disciplinas estratégicas, y entre personas que habían trabajado previamente en otras universidades o en empresas. El estudio de Araújo y otros (2017), mencionado antes, encontraba no solo que las mujeres suelen colaborar con equipos más igualitarios en términos de sexos, sino que también, por lo menos en las Ciencias Naturales, presentan una mayor inclinación a colaborar con personas de otros ámbitos de conocimiento. Retomando el trabajo de Van Rijnsoever y Hessels (2011), Woolley y otros (2014), no encontraban que el género influyera en la inclinación a la investigación interdisciplinaria en el personal docente e investigador de Ciencias Sociales australiano, aunque sí la inclinación hacia la investigación aplicada, o la diversidad de la experiencia laboral previa.

A pesar de que los resultados de la investigación no siempre sean concluyentes, el género parece tener cierta influencia en muchos fenómenos relativos a la comunicación, producción e impacto científico, como la revisión por pares, la composición de los comités editoriales de revistas, los patrones de colaboración e incluso la citación del trabajo de investigación. La tendencia, de la que hablábamos al principio, a enfatizar indicadores de impacto a nivel individual podría influir en la brecha entre hombres y mujeres, incluso cuando los indicadores se recaben de los medios sociales. El objetivo del presente trabajo es describir la presencia y la actividad en algunos medios sociales del personal docente e investigador en Ciencias de la Documentación en España. Concretamente, se analizará la presencia, actividad y visibilidad del personal docente e investigador perteneciente a los departamentos de Biblioteconomía y Documentación de las Universidades españolas en las siguientes plataformas: Google Scholar Citations (GSC), ResearchGate (RG), y Twitter. 


\subsection{Los medios sociales en la comunicación científica}

La participación de la comunidad científica en los medios sociales se contextualiza en la que Wildgaard y otros (2014), citando a Whitley, denominan "economía de la reputación". La reputación científica, según este equipo de investigación, que se centra en indicadores tradicionales, se manifestaría por encima de todo en el curriculum investigador. Sin embargo, dada la popularidad de ciertas métricas procedentes de los medios sociales, cabe preguntarse por las características de la comunicación científica en estos, los procesos de producción de métricas como el RGScore (Copiello y Bonifaci, 2018) y, en el caso que nos concierne, sobre posibles diferencias de género. Ward, Bejarano y Dudás (2015) denominan los perfiles académicos con la expresión de "selfies académi$\cos ^{\prime \prime}$, destacando varias funciones en el sistema de producción y comunicación de conocimiento, entre otras la de comunicar, visibilizar la actividad científica realizada, debatir y realizar actividades de networking. Unos perfiles activos en los medios sociales aumentan la visibilidad del trabajo investigador a la vez que ayudan a recoger métricas de impacto alternativas a los indicadores bibliométricos tradicionales (Bik y Goldstein, 2013). Los perfiles sociales son auténticas autopresentaciones del personal de investigación que acaba siendo juzgado y valorado por su trabajo y por las métricas que cada plataforma pueda proveer, pero también por su apariencia y forma de proponerse. Tsou y otros (2016) analizan la forma de presentarse y la manera en la que se les percibe de un conjunto de perfiles académicos, prestando especial atención a tres variables, en concreto, género, raza y edad, en tres plataformas: Microsoft Academic Search, Google Scholar y Mendeley. Clasificando a través del servicio de Amazon Mechanical Turk más de 10.000 perfiles académicos, encuentran que las mujeres, especialmente las más jóvenes, se percibían como las menos profesionales en el conjunto analizado, aunque puntuaban por encima de los demás en cuanto a atractivo. Más allá de la autopresentación, participar en la web social aportaría al quehacer académico tradicional importantes novedades, como la posibilidad de intercambiar productos académicos diferentes al artículo de investigación, como código, datos o resultados negativos de la investigación, o revelar al gran público por lo menos algunos procesos de investigación (Ward y otros, 2015). Zhu y Purdam (2017), centrándose en los perfiles del personal académico que denominan "super-usuarios", constatan que los medios sociales se utilizan principalmente para comunicar novedades y avances de la investigación propia. El networking es sin duda otra razón importan- te para estar en los medios sociales junto con la mayor visibilidad que estos pueden brindar para el trabajo investigador (Rinaldi, 2014). Otras razones para tener una presencia en la web incluyen la adquisición de conocimiento especializado o formar parte de una comunidad de pares junto con la autopromoción, según destacan Meishar-Tal y Pieterse (2017) en un estudio con personal docente e investigador de tres instituciones académicas israelíes, siendo la interacción con los pares la actividad menos destacada. La necesidad de establecer contactos prima sobre el entretenimiento y la búsqueda de información entre las razones para consumir blogs científicos de un conjunto de casi 3000 lectores y lectoras de blogs científicos (Brown Jarreau y Porter, 2017).

Collins, Shiffman, y Rock (2016) subrayan las posibilidades de comunicación bidireccional de los medios sociales que, por eso, podrían utilizarse para comunicar con la ciudadanía. Los resultados de la encuesta internacional que realizan con personal científico activo en los medios sociales por razones profesionales, sin embargo, sitúan la comunicación con el gran público a través de Twitter en un segundo lugar después de la comunicación con pares. Las actividades de interacción con la ciudadanía no pasan necesariamente por Twitter, y Bik y Goldstein (2013) aluden a los blogs como herramientas poderosas de comunicación con el público y a las iniciativas de ciencia ciudadana como www.scistarter.com, una plataforma que permite a toda persona interesada seleccionar un proyecto de investigación y participar en él. Esta línea de investigación sobre el uso de los medios sociales para fines académicos y de comunicación con el gran público podría, según Kjellberg y otros (2016), ayudar a comprender cómo los medios sociales contribuyen a crear un clima de confianza en la ciencia, en el personal de investigación y en su trabajo. Por otro lado, según algunas posturas, los medios sociales pueden aportar mayor transparencia y accesibilidad, pero también mayor vulnerabilidad, según demuestran algunos casos de científicos acosados por los medios.

\subsection{Patrones de uso de los medios sociales en la comunidad académica}

Normalmente, el uso de los medios sociales en la comunicación científica se ha relacionado con el estatus académico y la edad, siendo más proclives a utilizar las redes sociales las personas más jóvenes o con un estatus académico más afianzado, aunque, en general, la comunidad académica en el conjunto resulta más bien reacia al uso de la web social. El estudio de Mas-Bleda y otros (2014), por ejemplo, ha contabilizado la presencia 
en la web social de un conjunto de investigadores europeos muy citados en Web of Science, es decir, con un impacto probado según los sistemas tradicionales. El estudio encontró que, aunque la mayoría de las personas de la muestra tenía algún tipo de presencia en la web normalmente a través de sitios web personales o institucionales, la participación en el conjunto de plataformas sociales seleccionadas (Google Scholar, Mendeley, Academia, LinkedIn, SlideShare) era más bien escasa, pues solo el $28 \%$ utilizaba alguna de ellas, centrándose principalmente en la red social LinkedIn. A pesar de la participación limitada de la comunidad científica en la web social, reiterada por Mas-Bleda y Aguillo (2015), que mencionan varias encuestas internacionales sobre el tema, existen expectativas de que vaya creciendo a medida que las nuevas generaciones reemplacen a las viejas. En una perspectiva longitudinal, Ortega (2017) encuentra que la repartición por categoría profesional de los perfiles correspondientes a la plantilla del CSIC en Academia.edu, ResearchGate y Google Scholar Citations, ha ido definiéndose de forma coherente a su distribución real en el periodo abril de 2014- septiembre de 2015.

En general, sin embargo, los resultados en cuanto a la edad como predictor del uso de los medios sociales son más bien contradictorios, pues en algunos no se encuentra relación entre la edad y la inclinación a utilizar los medios sociales (Mikki y otros, 2015), mientras que en otros serían precisamente los más jóvenes los más reacios a participar en la web social. Nicholas y otros (2017) encuentran más bien una escasa propensión en 116 personas al principio de la carrera académica para aprovechar las potencialidades de los medios sociales, la ciencia abierta y el acceso abierto, debido a las condiciones precarias de trabajo y a la presión por publicar en revistas de alto impacto. Además de la vulnerabilidad a la que exponen los medios sociales, la investigación ha destacado otras razones del escaso uso de los mismos, entre otras la falta de tiempo y el desconocimiento de cómo funcionan (Donelan, 2016), por lo menos en las disciplinas STEM (ciencia, tecnología, ingeniería y matemáticas). En concreto, con respecto a Twitter, los participantes en la encuesta de Collins y otros (2016) indican la falta de conocimiento de Twitter, no solo desde un punto de vista técnico, sino también de lo que permite realizar, en general, para la comunicación científica o dentro del aula. A la falta de tiempo y el desconocimiento de las funcionalidades de varias plataformas, Greifeneder y otros (2018), en una perspectiva cualitativa, añaden el escepticismo de la comunidad académica con respecto a las redes sociales sobre todo entre el personal aca- démico más joven o en posiciones intermedias en la jerarquía universitaria. Según Rinaldi (2014), la razón principal del escaso éxito de los medios sociales en la comunidad académica sería que no cambian sustancialmente la forma de trabajar en el día a día y, sobre todo, no afecta a los criterios de evaluación del trabajo investigador. Entre otras barreras al uso de los medios sociales, Zhu y Purdam (2017) mencionan las preocupaciones relativas a la ausencia de claridad y políticas de la ciencia abierta, los riesgos relativos a la propiedad intelectual, los riesgos implícitos en la reutilización de los datos y el riesgo de comunicar ciencia a un público de personas que carece de los conocimientos técnicos y especializados necesarios para comprenderla. Existiría un riesgo de simplificación que apunta a que no basta con publicar, sino que es necesario interactuar. Para resumir, utilizando una clasificación de Donelan (2016), los obstáculos al uso de los medios sociales se pueden diferenciar en funcionales relacionados con la percepción de no poseer las habilidades y el tiempo necesario para invertir en estas herramientas - y psicológicos, relacionados con una visión negativa de los medios sociales.

Además de las diferencias de género, también están documentadas las diferencias disciplinarias con respecto al uso de las redes sociales, aunque de forma dispersa. Holmeberg y Thelwall (2014) analizan las diferencias disciplinarias en el uso de Twitter. Si en el conjunto los investigadores seleccionados compartían más links que el público típico de Twitter, el uso de la plataforma variaba según la disciplina. En Bioquímica, por ejemplo, se apreciaba una mayor inclinación para el retweet, mientras que en las Humanidades y en las Ciencias Cognitivas se daban más conversaciones, y en Economía se compartían más links. Desde el punto de vista de la actividad, los perfiles correspondientes a las grandes áreas de conocimiento presentaban además otras diferencias, pues si los de Biología y Biomedicina resultaban escasamente activos, los de Ciencias Naturales eran muy activos, sobre todo en actividades como ojear artículos y conectar con otros perfiles. Ortega (2017), analizando longitudinalmente la presencia del personal de investigación del CSIC en Academia. edu, ResearchGate y Google Scholar Citations, encuentra que es posible hablar de un uso disciplinario diferencial de las tres plataformas, pues si en Academia.edu la participación de las Humanidades y Ciencias Sociales es más destacada, los perfiles de Google Scholar Citations corresponden mayoritariamente a las Ciencias Físicas y Naturales, mientras que en ResearchGate es posible apreciar el predominio de la Biología y la Biomedicina, reiterando los resultados de un trabajo pre- 
vio (Ortega, 2015). Costas y otros (2017) analizan por disciplina la presencia de investigadores con artículos en WoS y que hayan tweeteado por lo menos un artículo de investigación y encuentran que las Ciencias Sociales y las Humanidades están más presentes, siendo las Ciencias Naturales las que menos. Resultados parcialmente parecidos hallan Ke y otros (2017), quienes constatan en una muestra de perfiles académicos en Twitter una hiperrepresentación de las Ciencias Sociales y la Informática respecto a las Matemáticas, la Física y las Ciencias Naturales.

\section{METODOLOGÍA}

Con el objetivo indicado anteriormente, se recabaron algunos datos de tres plataformas sociales, dos específicas del ámbito académico, Google Scholar Citations y ResearchGate, y otra más general, Twitter, para describir y comparar el personal docente e investigador femenino y masculino en Documentación en términos de presencia, actividad, visibilidad e interdisciplinariedad.

\subsection{Población estudiada}

El conjunto de profesoras y profesores cuya presencia y actividad fue objeto de estudio está reflejado en la Tabla I. La información relativa a las universidades que ofertan formación en Biblioteconomía y Documentación se obtuvo del sitio de la Federación Española de Asociaciones de Archiveros, Bibliotecarios, Arqueólogos, Museólogos y Documentalistas ( $A N A B A D$ ), donde se identificaron 16 , de las cuales se eligieron las 13 de mayor prestigio y tradición (Tabla I).

\subsection{Variables estudiadas}

Cada uno de los cuatro apartados de análisis identificados (presencia, actividad, visibilidad e interdisciplinariedad) se midió a través de diferentes variables, según se explica en lo que sigue.

\subsubsection{Presencia}

Para describir la presencia del colectivo estudiado en los medios sociales se contabilizaron los perfiles registrados en las tres plataformas en el periodo entre abril y junio de 2018. La identificación de perfiles en Twitter presenta unas dificultades añadidas respecto a RG y GSC, que son plataformas de uso exclusivamente académico. Al tratarse de una red social generalista, existen problemas de homonimia, pero, además, su carácter, en cierta medida informal, hace que muchas personas utilicen versiones más coloquiales o abreviadas de su nombre, dificultando aún más la tarea. Por otro lado, atendiendo a la red de personas seguidoras o a las que se sigue, ha sido posible identificar con mayor seguridad aquellos perfiles que planteaban dudas.

Tabla I. Distribución por sexo y universidad de la población estudiada. Orden descendente según el total del profesorado. (M=Mujeres, $\mathrm{H}=$ Hombres)

\begin{tabular}{|l|c|c|c|}
\hline Universidad & M & H & Total \\
\hline Universidad de Barcelona & 32 & 36 & 68 \\
\hline Universidad Complutense de Madrid & 26 & 19 & 45 \\
\hline Universidad Carlos III de Madrid & 22 & 19 & 41 \\
\hline Universidad de Granada & 15 & 20 & 35 \\
\hline Universidad de Zaragoza & 16 & 14 & 30 \\
\hline Universidad de Valencia & 10 & 17 & 27 \\
\hline Universidad de Salamanca & 12 & 9 & 21 \\
\hline Universidad de Murcia & 9 & 12 & 21 \\
\hline Universidad de Extremadura & 11 & 7 & 18 \\
\hline Universitat Oberta de Catalunya & 7 & 9 & 16 \\
\hline Universidad de León & 9 & 2 & 11 \\
\hline Universidad de Alcalá & 8 & 2 & 10 \\
\hline Universidad de La Coruña & 6 & 0 & 6 \\
\hline Total & $\mathbf{1 8 3}$ & $\mathbf{1 6 6}$ & $\mathbf{3 4 9}$ \\
\hline
\end{tabular}




\subsubsection{Actividad}

La actividad se cuantificó a través de datos recabados de RG y Twitter. RG permite recopilar los trabajos científicos realizados y adjuntar su respectivo texto completo. Además, permite interactuar con otras personas registradas participando en foros, tanto a través de preguntas lanzadas a la comunidad como de respuestas ofrecidas a las mismas. Esta actividad de participación queda recogida en un indicador de impacto que ofrece la plataforma, el RG Score, en términos porcentuales (Orduña-Malea y otros, 2016a; Orduña-Malea y otros, 2017), y se recogió como tal. El RG score se calcula teniendo en cuenta 4 aspectos, cada uno de los cuales tiene un peso porcentual: las publicaciones de cada perfil, sus seguidores, las preguntas y las respuestas a la comunidad. En resumen, se anotó el número de documentos subidos a la plataforma para cada perfil, así como el peso en términos porcentuales de las preguntas y respuestas en el cómputo del RG score.

Por otro lado, en Twitter, se clasificaron los perfiles encontrados en base a la tipología desarrollada por Barbour y Marshall (2012), quienes caracterizan cinco estilos diferentes de "personas" académicas en los medios sociales, y teniendo en cuenta asimismo la base conceptual de la clasificación de Nentwich y König (2014), quienes enfatizan más bien el tipo de actividad y la intensidad de uso. La tipología identificada en Barbour y Marshall (2012), y que está reflejada en la Tabla II, se ha ajustado a la actividad del colectivo de Documentación en Twitter teniendo en cuenta el trabajo previo de Álvarez-Bornstein y Montesi (2016). En esta etnografía virtual de un colectivo de 26 personas del área de la Documentación, se caracterizaba su actividad en Twitter en cuatro grandes líneas, enfatizando la difusión de contenidos tanto de carácter científico como profesional o de otra índole. Para eso añadimos un sexto perfil a los de Barbour y Marshall (2012) que hemos denominado "difusor" y que denota una comunicación de tipo unidireccional en la que se da difusión a información procedente de instituciones como bibliotecas, universidades, colegios profesionales y similares, o recursos, más que difundir información sobre la actividad propia o de otras personas.

La clasificación se realizó atendiendo a la actividad más reciente desempeñada en los diferentes perfiles. Adicionalmente, a través de la aplicación Twitonomy (www.twitonomy.com), se recabaron los siguientes datos de actividad complementarios para el año anterior a la recogida de los mismos (del 1/07/2017 al 30/06/2018): a) número de tweets, b) porcentaje de respuestas y c) porcentaje de retweets sobre el total de tweets enviados, d) número de menciones, e) número de links y f) porcentaje de tweets publicados por cada perfil que se han reenviado por parte de otros perfiles.

Tabla II. Clases de perfiles de Twitter en base a la actividad desarrollada según (Barbour y Marshall, 2012) y (Álvarez Bornstein y Montesi, 2016)

\begin{tabular}{|l|l|}
\hline Perfil formal & $\begin{array}{l}\text { Perfil simple, caracterizado por la ausencia de interactividad y una comunicación } \\
\text { fundamentalmente unidireccional, parecido a un sitio web. Se trata de un perfil "fijo" que no } \\
\text { está abierto a comentarios, ideas y feedback del exterior. }\end{array}$ \\
\hline Perfil difusor & $\begin{array}{l}\text { Difunde mucha información institucional (bibliotecas, Wikipedia, museos, medios de } \\
\text { comunicación...) o de interés profesional. Los retweets persiguen principalmente difundir la } \\
\text { información. }\end{array}$ \\
\hline Perfil conectado & $\begin{array}{l}\text { Se centra en compartir ideas y en crear redes de contactos. Presenta un alto nivel de } \\
\text { interactividad y conexión con contenidos externos a la plataforma (blogs o artículos). Interactúa } \\
\text { con "micro-públicos" y construye pequeñas redes. Enlaza diferentes perfiles/plataformas que } \\
\text { tienen que ver con su actividad: por ejemplo, remite a su última actualización del blog desde } \\
\text { Twitter. }\end{array}$ \\
\hline Perfil completo & \begin{tabular}{l} 
No hay frontera entre lo profesional y lo personal. \\
\hline Perfil docente
\end{tabular} \\
\hline Inactivo & $\begin{array}{l}\text { En lugar de dirigirse a pares o colegas, se dirige y utiliza el perfil para conectar con el alumnado } \\
\text { real o potencial (la generación de nativos digitales), funcionando como una extensión del } \\
\text { campus virtual. }\end{array}$ \\
\hline
\end{tabular}




\subsection{Visibilidad}

La propia existencia de un perfil puede considerarse como un elemento que confiere visibilidad al personal docente. Sin embargo, en este apartado aportamos también otros datos. En concreto, para RG: a) el porcentaje de documentos en acceso abierto respecto al total de los mismos para cada perfil, b) el porcentaje de documentos diferentes a los tradicionales (article, book, chapter, conference paper) sobre el total de documentos subidos a la plataforma, c) el total de lecturas (reads) por perfil, y d) el promedio de lecturas (reads) por ítem en RG a julio de 2018. Hay que tener en cuenta que el total de lecturas depende de la antigüedad de cada perfil, dato que no se ha contabilizado en el presente estudio. Entendimos que la tipología de documentos diferentes a los tradicionales disponibles en RG sería un indicador de visibilidad, poniendo de manifiesto productos científicos menos visibles de la actividad investigadora. Para Twitter, se recopilaron: e) el número de seguidores/seguidoras por perfil, y f) el número de listas de Twitter en las que aparecen los perfiles analizados.

\subsection{Interdisciplinariedad}

Se ha medido la forma en la que las personas con perfil en RG y GSC perciben su especialización. Los medios sociales académicos permiten, entre otras cosas, auto-presentar la actividad desempeñada y es interesante saber si existen diferentes maneras de percibir la propia actividad investigadora entre hombres y mujeres. Para eso se contabilizó el número de especialidades registradas en los perfiles de RG (en el apartado Skills and expertise) y las especialidades de GSC. En esta última plataforma solo son visibles las primeras 5 opciones.

Las dimensiones de actividad y visibilidad no se analizaron en GSC. Las posibilidades de actividad en esta plataforma son mínimas, puesto que el contenido de cada perfil se conforma de manera semi-automática y se admiten posibilidades de edición escasas, como por ejemplo la rectificación de errores. En cuanto a la visibilidad, la plataforma refleja más bien indicadores de impacto que de visibilidad basados en citas.

Los datos recopilados en los diferentes apartados se presentan a través de estadísticas descriptivas para profesoras y profesores. La hipótesis del estudio es la de la existencia de diferencias entre hombres y mujeres en las variables seleccionadas $y$, para este fin, donde ha sido procedente se ha realizado la prueba $U$ de Mann-Whitney para muestras independientes con el software SPSS versión 25. La elección de este test no paramétrico se debe a que los datos recogidos presentan distribuciones asimétricas.

\section{RESULTADOS}

\subsection{Presencia en RG, GSC y Twitter}

La presencia de profesores y profesoras es muy igualada en las tres plataformas, aunque hay una mayor proporción de profesores que profesoras con presencia en las mismas, especialmente en GSC. Destaca RG como la plataforma con mayor presencia del colectivo estudiado y Twitter como la de menor presencia (Tabla III).

\subsection{Actividad}

La actividad desempeñada en RG muestra, por un lado, que los dos conjuntos difieren en el volumen de documentos subidos a la plataforma (Tabla IV), siendo esta diferencia significativa (prueba $U$ de Mann-Whitney: $U=2865,5$, $p$ valor $=0,000228$ ) y posiblemente reflejando la disparidad en términos de producción apreciada en la literatura previa. En esta plataforma, la población objeto de estudio no interactúa con la comunidad, a tenor de los indicadores analizados, es decir, el peso que corresponde a preguntas y respuestas en el RG Score. De hecho, muy pocas personas tienen reconocida su participación en los debates de la plataforma en el cómputo de su RG Score (Tabla IV).

Tabla III. Presencia de la población estudiada en RG, GSC y Twitter (M=Mujeres, H=Hombres)

\begin{tabular}{|c|c|c|c|c|c|c|c|}
\hline & $\begin{array}{c}\text { Total } \\
\text { muestra }\end{array}$ & $\begin{array}{c}\text { Con perfil } \\
\text { en RG }\end{array}$ & $\begin{array}{l}\text { \% sobre } \\
\text { el total }\end{array}$ & $\begin{array}{c}\text { Con perfil } \\
\text { en GSC }\end{array}$ & $\begin{array}{c}\% \text { sobre el } \\
\text { total }\end{array}$ & $\begin{array}{l}\text { Con perfil } \\
\text { en Twitter }\end{array}$ & $\begin{array}{c}\text { \% sobre el } \\
\text { total }\end{array}$ \\
\hline$M$ & 183 & 92 & $50,3 \%$ & 79 & $43,2 \%$ & 63 & $34,4 \%$ \\
\hline $\mathrm{H}$ & 166 & 91 & $54,8 \%$ & 83 & $50 \%$ & 64 & $38,5 \%$ \\
\hline Total & 349 & 183 & $52,4 \%$ & 162 & $46,4 \%$ & 127 & $36,4 \%$ \\
\hline
\end{tabular}


Tabla IV. Dimensiones de la actividad en RG ( $M=$ Mujeres, $\mathrm{H}=$ Hombres)

\begin{tabular}{|l|c|c|}
\hline \multicolumn{2}{|l|}{} & M \\
\hline Documentos subidos a la plataforma & 37,2 & 63,8 \\
\hline Promedio & 37,2 & 58,2 \\
\hline Desv. Est. & 28 & 42 \\
\hline Mediana & 31.5 & 53 \\
\hline Rango Inter. & 5 & 7 \\
\hline Perfiles con por lo menos una respuesta/pregunta \\
\hline \multicolumn{2}{|c|}{} \\
\hline
\end{tabular}

La clasificación de los perfiles de Twitter según el tipo de actividad desempeñada reitera solo parcialmente la escasa participación de la comunidad académica en los medios sociales, mientras que pone de manifiesto diferencias entre hombres y mujeres, según puede verse en la Figura 1. Las mujeres presentan más del doble de perfiles inactivos o bloqueados en Twitter, separan más que los hombres la vida personal de la profesional (perfil completo, $27 \%$ vs $37 \%$ ) y presentan una inclinación menos acentuada a difundir sus méritos por los medios sociales (perfil formal, $5 \%$ vs $19 \%)$. También tienden a desempeñar actividades de difusión informativa más a menudo que los hombres (perfil difusor, $11 \%$ vs $5 \%$ ), e interactúan menos con otras personas (perfil conectado, $9 \%$ vs $16 \%$ ).

Las otras dimensiones de la actividad en Twitter reflejadas en la Tabla $V$ no presentan grandes diferencias entre los dos conjuntos, excepto por unos casos. Destaca el volumen considerablemente superior del promedio de tweets publicados en el pe- riodo estudiado por el colectivo de profesores, aunque los valores atípicos son más comunes en este colectivo según se aprecia por las respectivas desviaciones estándar de la media (Tabla V). Por otro lado, se aprecian diferencias menores si tenemos en cuenta la mediana en lugar de la media (mediana profesoras $=69$, mediana profesores $=63$ ). El porcentaje de respuestas (replies) y retweets sobre el total de los tweets publicados así como el número de menciones por tweet es ligeramente superior en el colectivo de profesores, apuntando a una mayor inclinación a interactuar con otros perfiles en esta plataforma, aunque no de forma significativa.

En efecto, la hipótesis nula de igualdad entre hombres y mujeres pudo rechazarse solo en el caso del número de links por tweet $(p=0,015, U$ de Mann-Whitney $=1448,5$ ). Esto apunta a que los tweets de los profesores tienden a remitir más a menudo a contenidos web complementarios a través de links respecto a los de las profesoras.

Figura 1. Tipo de actividad de perfiles de Twitter para el profesorado de Documentación analizado
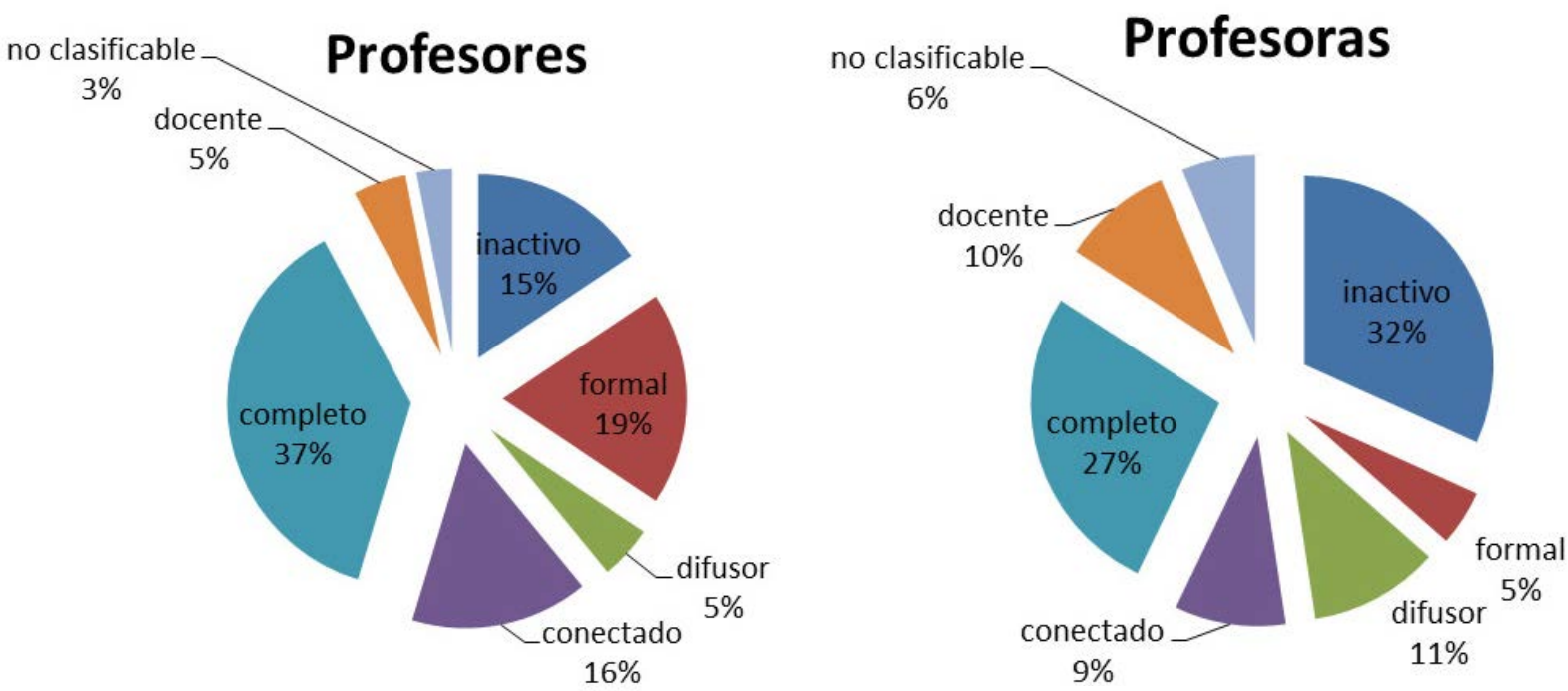
Tabla V. Estadísticas descriptivas relativas a diferentes dimensiones de la actividad en Twitter ( $M=$ Mujeres, $\mathrm{H}=\mathrm{Hombres}$ )

\begin{tabular}{|c|c|c|}
\hline Twitter & M & $\mathbf{H}$ \\
\hline \multicolumn{3}{|c|}{ No tweets del $01 / 07 / 2017$ al $30 / 06 / 2018$} \\
\hline Promedio & 268,24 & 592,77 \\
\hline Desv. Est. & 453,8 & 1758,3 \\
\hline Mediana & 69 & 63 \\
\hline Rango Inter. & 376 & 553 \\
\hline \multicolumn{3}{|l|}{$\%$ de replies } \\
\hline Promedio & 7,58 & 9,23 \\
\hline Desv. Est. & 16,29 & 16,91 \\
\hline Mediana & 1 & 4 \\
\hline Rango Inter. & 8.5 & 10 \\
\hline \multicolumn{3}{|l|}{$\%$ de retweets } \\
\hline Promedio & 43,25 & 46,16 \\
\hline Desv. Est. & 39,79 & 36,97 \\
\hline Mediana & 44,5 & 49,5 \\
\hline Rango Inter. & 84,7 & 78,5 \\
\hline \multicolumn{3}{|c|}{ User mentions por tweet } \\
\hline Promedio & 0,25 & 0,36 \\
\hline Desv. Est. & 0,37 & 0,46 \\
\hline Mediana & 0,09 & 0,2 \\
\hline Rango Inter. & 0,41 & 0,6 \\
\hline \multicolumn{3}{|l|}{ Links por tweet } \\
\hline Promedio & 0,10 & 0,23 \\
\hline Desv. Est. & 0,18 & 0,29 \\
\hline Mediana & 0,02 & 0,09 \\
\hline Rango Inter. & 0,14 & 0,32 \\
\hline
\end{tabular}

\subsection{Visibilidad}

Las dimensiones de la visibilidad analizadas en RG ponen de manifiesto diferencias derivadas en parte de las que se han expuesto en términos de actividad. Mientras que las diferencias en el promedio de reads por ítem entre hombres y mujeres no son significativas (U de Mann-Whitney=3705, p valor = 0,224), sí lo es la diferencia entre el promedio de reads totales para los dos conjuntos (U de Mann-Whitney= 2948, $\mathrm{p}$ valor $=0,001)$, posiblemente derivada de las disparidades en términos de documentos subidos a la plataforma que veíamos antes. La disponibilidad de documentos en acceso abierto para perfiles masculinos y femeninos presenta diferencias en términos promedios, pero no significativas.

En cuanto al porcentaje de documentos tradicionales sobre el total, los dos conjuntos presentan distribuciones diferentes, aunque ambas tengan un sesgo positivo, pues, mientras que los valores de las mujeres se concentran alrededor del 100\%, los de los hombres se distribuyen mayoritariamente entre $90 \%$ y $100 \%$ (Figura 2 ). Con lo cual es posible apreciar una mayor disposición a publicar productos científicos diferentes a los tradicionales por parte de los profesores frente a las profesoras ( $U$ de Mann-Whitney $=3780,5$, p valor $=0.017$ ) .
En cuanto a la visibilidad dentro de la comunidad científica y profesional de Twitter, en cambio, hombres y mujeres presentan diferencias importantes, cuando se mide el número medio de seguidores/ seguidoras en los perfiles analizados, según puede verse en la Tabla VII. El total de seguidores de los perfiles masculinos prácticamente dobla al de los perfiles femeninos (49469 vs 25544), siendo el número de perfiles analizados muy parecido (64 vs 63). La imagen que transmiten estos datos es de una visibilidad claramente más marcada de los hombres que, además, buscan mayores conexiones en la comunidad profesional siguiendo de media a un número superior de perfiles y aparecen con más frecuencia que las mujeres en listas. Las diferencias en el promedio de seguidores y de listas para hombres y mujeres son, además, significativas (Seguidores: $U$ de Mann-Whitney $=1357$, p valor $=0,001$; Listas: $U$ de Mann-Whitney $=1359$, $p$ valor $=0,005$ ). Presenta diferencias significativas también la última dimensión analizada, el porcentaje de tweets del perfil retweeteados por otros perfiles ( $U$ de Mann-Whitney = 1364, $p=0,007$ ), apuntando a que los tweets de los profesores tienden a retweetearse con más frecuencia. Una vez más la actividad en los medios sociales parece indicar un mayor impacto o alcance de la producción masculina frente a la femenina. 
Tabla VI. Dimensiones de la visibilidad en RG ( $M=$ Mujeres, $\mathrm{H}=$ Hombres)

\begin{tabular}{|c|c|c|}
\hline RG & M & $\mathbf{H}$ \\
\hline \multicolumn{3}{|c|}{$\%$ de documentos en abierto sobre el total } \\
\hline Promedio & 54,5 & 60,0 \\
\hline Desv. Est. & 24,6 & 22,4 \\
\hline Mediana & 60,0 & 63,6 \\
\hline Rango Inter. & 33,4 & 28,9 \\
\hline \multicolumn{3}{|c|}{$\%$ de documentos tradicionales sobre el total } \\
\hline Promedio & 96,4 & 95,4 \\
\hline Desv. Est. & 7,0 & 6,7 \\
\hline Mediana & 100 & 97,1 \\
\hline Rango Inter. & 4,65 & 6,76 \\
\hline \multicolumn{3}{|l|}{ Reads por item } \\
\hline Promedio & 141,7 & 194,6 \\
\hline Desv. Est. & 271,9 & 537,3 \\
\hline Mediana & 37,2 & 47,6 \\
\hline Rango Inter. & 113,18 & 99 \\
\hline \multicolumn{3}{|c|}{ Reads por perfil } \\
\hline Promedio & 2163,2 & 5187 \\
\hline Desv. Est. & 2957,9 & 11055 \\
\hline Mediana & 1122 & 2228 \\
\hline Rango Inter. & 2202,7 & 5869,5 \\
\hline
\end{tabular}

Figura 2. Histogramas mostrando el porcentaje de documentos tradicionales sobre el total de los subidos a RG para profesoras y profesores. En el eje de las ordenadas se muestran las frecuencias absolutas de profesores y profesoras

Mujeres

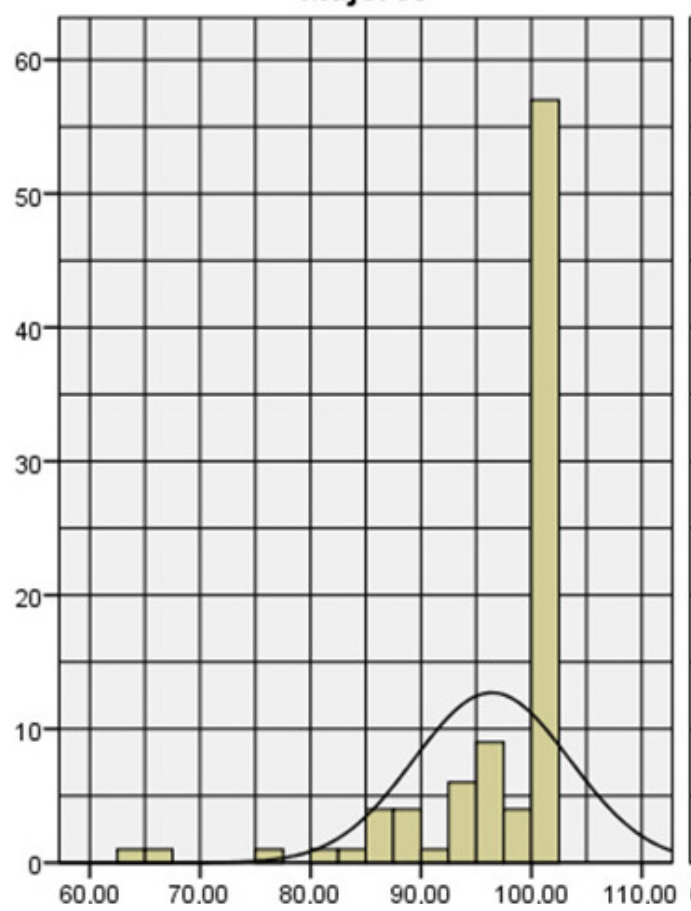

Hombres

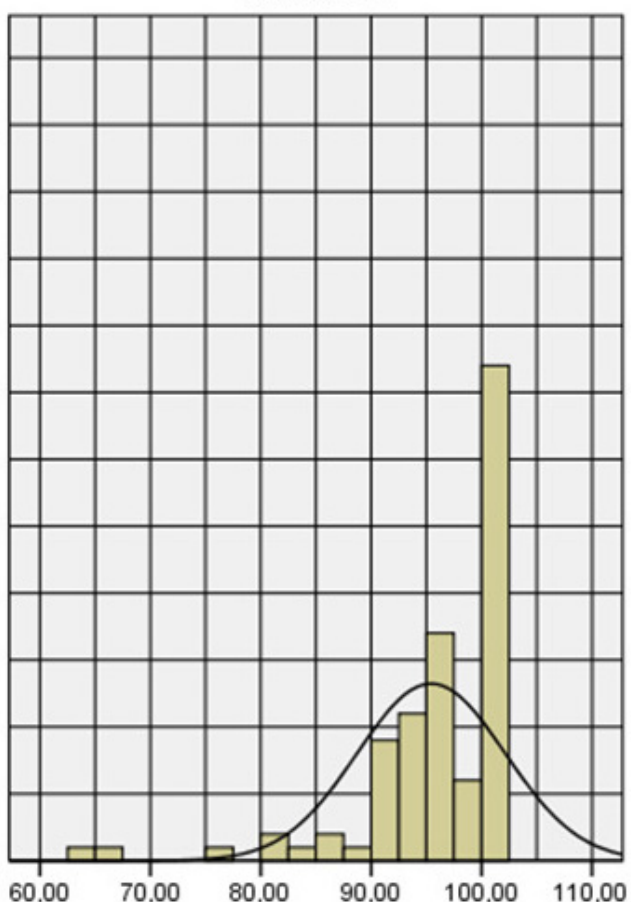


Tabla VII. Dimensiones de la visibilidad en Twitter ( $M=$ Mujeres, $\mathrm{H}=$ Hombres)

\begin{tabular}{|c|c|c|}
\hline Twitter & M & $\mathbf{H}$ \\
\hline \multicolumn{3}{|l|}{ Listas } \\
\hline Promedio & 26,4 & 58,8 \\
\hline Desv.Est. & 60,1 & 124,9 \\
\hline Mediana & 4 & 17 \\
\hline Rango Inter. & 27,7 & 49 \\
\hline \multicolumn{3}{|l|}{ Seguidores } \\
\hline Promedio & 405,4 & 772,9 \\
\hline Desv.Est. & 1058 & 1337,4 \\
\hline Mediana & 70 & 305 \\
\hline Rango Inter. & 320 & 791,5 \\
\hline \multicolumn{3}{|l|}{ Siguiendo } \\
\hline Promedio & 273,8 & 458,5 \\
\hline Desv.Est. & 445,2 & 776,9 \\
\hline Mediana & 108 & 195 \\
\hline Rango Inter. & 261 & 524 \\
\hline \multicolumn{3}{|c|}{$\%$ tweets retweeteados } \\
\hline Promedio & 7 & 14,4 \\
\hline Desv. Est. & 11,5 & 17,6 \\
\hline Mediana & 2 & 8,3 \\
\hline Rango Inter. & 9,3 & 21,7 \\
\hline
\end{tabular}

\subsection{Interdisciplinariedad}

No se aprecian grandes diferencias entre hombres y mujeres en cuanto a la percepción de la interdisciplinariedad en RG, pues los valores medios relativos al número de especialidades indicadas en los perfiles son muy parecidos (promedio 12,3 y 12,9 , mediana 9 y 9 para mujeres y hombres respectivamente). Se aprecian diferencias más claras

Tabla VIII. Número de especialidades reflejadas en GSC por sexo ( $M=$ Mujeres, $H=$ Hombres)

\begin{tabular}{c|c|c}
\hline Especialidades en GSC & $\mathbf{M}(\mathbf{\%})$ & $\mathbf{H}(\mathbf{\%})$ \\
\hline 0 & 17,7 & 2,4 \\
\hline 1 & 10,1 & 10,8 \\
\hline 2 & 10,1 & 4,8 \\
\hline 3 & 13,9 & 16,9 \\
\hline 4 & 16,5 & 27,7 \\
\hline 5 & 31,6 & 37,3 \\
\hline
\end{tabular}

en GSC donde los hombres tienden a presentarse como especialistas en un mayor número de áreas. Las profesoras superan notablemente a los hombres en el número de perfiles que no indican ninguna especialidad $(17,7 \%$ vs $2,4 \%)$, indicando un promedio de 3 especialidades frente a las 3,67 de los hombres. Las diferencias en este apartado son significativas ( $U$ de Mann-Whitney $U=2626$, $p$ valor $=0,024)$ (Tabla VIII).

\section{DISCUSIÓN Y CONCLUSIONES}

El análisis de diferentes dimensiones de la presencia, actividad, visibilidad y percepción de la actividad investigadora en términos de interdisciplinariedad del profesorado de Documentación adscrito a universidades españolas pone de manifiesto diferencias en el comportamiento de profesoras y profesores en los medios sociales. Por lo que concierne a la presencia en las plataformas estudiadas, RG, Twitter y GSC, en el conjunto, los resultados apuntan a que no existen diferencias importantes, independientemente de que se trate 
de redes sociales académicas como RG y GSC o de redes sociales generalistas como Twitter. Twitter y GSC presentan unos porcentajes ligeramente superiores de perfiles masculinos respecto a los femeninos, en línea con estudios previos realizados en otros contextos y citados en la introducción. Si consideramos que la presencia de perfiles de profesorado de Documentación en RG, GSC y Twitter asciende, en el conjunto y con independencia del sexo, respectivamente al $52,4 \%$, el 46,4 y el $36,4 \%$, se puede concluir que la presencia de perfiles de profesoras tiende a ser mayor en las plataformas con más perfiles de Documentación totales. Las diferencias más marcadas, aunque muy sutiles, se encuentran en GSC. Quizás una de las razones podría estar en que esta plataforma presenta, por cada perfil, indicadores bibliométricos tradicionales, basados en el nivel de citación, como el número total de citas o el índice $h, y$ además enfatiza el historial de publicación individual. Estudios previos han puesto de manifiesto diferencias entre profesorado femenino y masculino de Documentación en términos de producción, resultando los hombres más productivos, aunque no en términos de citación (Peñas y Willett, 2006). Las diferencias en términos de citación se han apreciado más marcadamente en áreas relacionadas como la Comunicación (Knobloch-Westerwick y Glynn, 2013). GSC, al enfatizar el portfolio de publicaciones y los respectivos indicadores, visibiliza públicamente posibles diferencias y desventajas, desalentando, en cierto sentido, al personal investigador menos productivo o cuya producción presenta menor impacto. Por otro lado, las métricas de RG, aunque el RG Score parece basarse en parte en indicadores tradicionales, como el factor de impacto de las revistas en las que se publica (Orduña-Malea y otros, 2017), son más diversificadas y no están ni consolidadas ni reconocidas en la comunidad académica, algo que podría configurar un entorno menos competitivo y más atractivo para el profesorado femenino.

En cuanto a la actividad desempeñada en Twitter, el colectivo femenino destaca frente al masculino por una menor tendencia a utilizarlo para dar a conocer méritos personales - algo que hemos conceptualizado como "perfil formal" en la clasificación de los perfiles de Twitter. La actividad de las mujeres en Twitter se caracterizaría además por una mayor inclinación a difundir noticias e información de otras entidades (perfil difusor, $11 \%$ vs $5 \%$ ), participando de manera menos pronunciada que los hombres en los intercambios de información personal con otras personas (perfil conectado, $9 \%$ vs $16 \%$ ). Se trataría de una actividad que se desempeña desde una posición de invisibilidad - concepto clave en la tradición de pensamiento feminista (Martínez Lirola, 2010; Martínez Lirola, 2011) - o, cuando menos, de menor visibilidad y en una perspectiva de mayor cooperación. La actividad del profesorado femenino en Twitter se caracteriza además por la mayor presencia de perfiles inactivos y la menor inclinación a mezclar vida profesional y privada (perfiles completos), algo que podría relacionarse con las necesidades de la conciliación familiar y la supuesta imposibilidad de conciliar un rol profesional y competente con otro atento a las necesidades familiares. Los perfiles masculinos, además, destacan frente a los femeninos por un mayor uso de enlaces a recursos web dentro de los tweets publicados, algo que podría considerarse como revelador de una mayor competencia en el uso de Twitter para fines de investigación, pues los tweets publicados por la comunidad científica destacarían frente a los del público general por un mayor uso de links (Holmberg y Thelwall, 2014). En relación al uso de las tecnologías de la información y comunicación, Kim (2010) habla de una menor auto-eficacia de las mujeres frente a los hombres, entendiendo la auto-eficacia como algo que se desarrolla en los procesos diferenciales de socialización y que sería "[...] 'a judgment of one's capability to accomplish a certain level of performance'" (p.605). Diferentes niveles de auto-eficacia podrían explicar además el mayor porcentaje de perfiles inactivos del colectivo de profesoras, caracterizándose como obstáculos funcionales, según la expresión de Donelan (2016), al uso de los medios sociales. En todo caso, otros estudios indican que el uso de las tecnologías de la información difiere entre hombres y mujeres, especialmente en los países desarrollados (Galyani Moghaddam, 2010), poniendo de manifiesto la necesidad de estudiar posibles diferencias en el uso de los medios sociales para la comunicación científica, en el caso concreto de Twitter.

La actividad en RG revela diferencias significativas en términos de producción entre hombres y mujeres, siendo el promedio de publicaciones subidas respectivamente 63,8 y 37,2 , aunque las publicaciones recopiladas en este portal no tienen que recoger necesariamente la producción total de cada perfil. Tanto el colectivo femenino como el masculino resultan prácticamente inactivos por lo que concierne a la participación en los debates de RG.

En RG, la visibilidad de los perfiles femeninos se ve afectada por las diferencias en términos de producción, pues si no hay diferencias significativas entre hombres y mujeres en el promedio de reads por documento, sí las hay en el promedio de reads por perfil, casi doblando los reads de los perfiles masculinos (5188) a los de los perfiles femeninos (2163). Además, en RG las profesoras presentan una menor inclinación a subir contenido científico diferente al tradicional - es decir, que no sean ar- 
tículos, artículos de conferencia, capítulos de libros o libros. En Twitter, desde el punto de vista de la visibilidad, se observan diferencias importantes entre hombres y mujeres, teniendo en cuenta al promedio de seguidores, 773 para los hombres y 405 para las mujeres, y el promedio de listas en las que aparecen los perfiles analizados (59 para los hombres y 26 para las mujeres). Las mujeres son también menos activas buscando contactos y posibles seguidores que deriven de estos - pues de media siguen a muchos menos perfiles que los hombres ( 274 vs 458). Finalmente, en Twitter, los tweets publicados por mujeres se retweetean significativamente menos que los de los hombres y su actividad en Twitter tiene, consiguientemente, un alcance menor, reiterando estudios previos y en particular García Nieto (2013). García Nieto (2013), tras analizar la presencia de información y opiniones de científicas y científicos en cinco diarios españoles de gran tirada y difusión, encontraba que solo el $12 \%$ de las opiniones recogidas de junio a octubre de 2012 en internet correspondía a científicas, mientras que el $88 \%$ correspondía a científicos.

En cuanto a la autopercepción de la actividad investigadora, hombres y mujeres apenas difieren en el número de especialidades recopiladas en RG, aunque presentan diferencias significativas en GSC, donde hay más perfiles femeninos en los que no figura la especialización de la persona. Estos resultados contrastan con las expectativas de menor especialización disciplinaria y mayor interdiscipli-

\section{REFERENCIAS}

Abramo, G.; D'Angelo, C. A.; Murgia, G. (2013). Gender differences in research collaboration. Journal of Informetrics, 7(4), 811-822. https://doi.org/10.1016/j. joi.2013.07.002

Álvarez Bornstein, B.; Montesi, M. (2016). La comunicación entre investigadores en Twitter. Una etnografía virtual en el ámbito de las ciencias de la documentación. Revista Española de Documentación Científica, 39(4), 8. https://doi.org/10.3989/redc.2016.4.1352

Araújo, E. B.; Araújo, N. A.; Moreira, A. A.; Herrmann, H. J.; Andrade Jr, J. S. (2017). Gender differences in scientific collaborations: Women are more egalitarian than men. PloS one, 12(5), e0176791. https://doi. org/10.1371/journal.pone.0176791

Barbour, K.; Marshall, D. (2012). The academic online: Constructing persona through the World Wide Web. First Monday, 17(9). https://doi.org/10.5210/ fm.v0i0.3969

Beaudry, C. ; Larivière, V. (2016). Which gender gap? Factors affecting researchers' scientific impact in science and medicine. Research Policy, 45(9), 1790-1817. https://doi.org/10.1016/j.respol.2016.05.009 nariedad de las mujeres, según los estudios mencionados en la introducción. Aunque este fenómeno pueda justificarse por la naturaleza interdisciplinaria de la Documentación, que afectaría de forma igual a profesores y profesoras, cabe preguntarse si la auto-percepción que se ha medido en este estudio se corresponde con criterios más objetivos como la clasificación por especialidades que los índices de citas permiten realizar en base a las revistas de publicación. En todo caso la descripción "más escueta" del ámbito de trabajo del personal femenino estudiado puede reflejar dinámicas sociales más generales, como las que describe García Nieto (2013), quien, analizando cómo se presentan los científicos y científicas en la prensa española durante cinco meses de 2012, aprecia una desproporción importante a favor de los científicos en el número de calificaciones y apreciaciones, así como en la naturaleza de las mismas.

En conclusión, los datos analizados demuestran, en sintonía con estudios previos, que el comportamiento de la comunidad científica de Documentación en los medios sociales resulta condicionado por las características de las diferentes plataformas, en términos de presencia, actividad y auto-presentación. Estas herramientas no son neutrales y moldean en cierta medida los procesos de la comunicación científica que permiten desempeñar. En relación con el sesgo de género, sería interesante que estudios futuros profundizaran en el uso diferencial de las mismas por parte de hombres y mujeres.

Bik, H. M.; Goldstein, M. C. (2013). An introduction to social media for scientists. PLoS biology, 11(4), e1001535. https://doi.org/10.1371/journal.pbio.1001535

Brown Jarreau, P.; Porter, L. (2017). Science in the social media age: profiles of science blog readers. Journalism \& Mass Communication Quarterly, 95(1), 142-168. https://doi.org/10.1177/1077699016685558

Collins, K.; Shiffman, D.; Rock, J. (2016). How are scientists using social media in the workplace?. PloS one, 11(10), e0162680. https://doi.org/10.1371/journal. pone. 0162680

Copiello, S.; Bonifaci, P. (2018). A few remarks on ResearchGate score and academic reputation. Scientometrics, 114(1), 301-306. https://doi.org/10.1007/ s11192-017-2582-9

Costas, R.; van Honk, J.; Franssen, T. (2017). Scholars on Twitter: who and how many are they? $16^{\text {th }}$ International Conference on Scientometrics and Informetrics, October 16-20, Wuhan, China. Disponible en: https:// arxiv.org/abs/1712.05667 [última consulta: 22-122018]. 
Donelan, H. (2016). Social media for professional development and networking opportunities in academia. Journal of Further and Higher Education, 40(5), 706-729. https://doi.org/10.1080/0309877X.2015.1014321

Galyani Moghaddam, G. (2010). Information technology and gender gap: toward a global view. The Electronic Library, 28(5), 722-733. https://doi. org/10.1108/02640471011081997

García Nieto, M. T. (2013). ¿Son invisibles las mujeres científicas? Estudios sobre el mensaje periodístico, 19, 783-792. https://doi.org/10.5209/rev_ESMP.2013. v19.42161

Ghiasi, G.; Harsh, M.; Schiffauerova, A. (2018). Inequality and collaboration patterns in Canadian nanotechnology: implications for pro-poor and gender-inclusive policy. Scientometrics, 115(2), 785-815. https://doi. org/10.1007/s11192-018-2701-2

Greifeneder, E.; Pontis, S.; Blandford, A.; Attalla, H.; Neal, D.; Schlebbe, K. (2018). Researchers' attitudes towards the use of social networking sites. Journal of Documentation, 74(1), 119-136. https://doi. org/10.1108/JD-04-2017-0051

Helmer, M.; Schottdorf, M.; Neef, A. ; Battaglia, D. (2017). Gender bias in scholarly peer review. Elife, 6, e21718. https://doi.org/10.7554/eLife.21718

Holmberg, K.; Thelwall, M. (2014). Disciplinary differences in Twitter scholarly communication. Scientometrics, 101(2), 1027-1042. https://doi.org/10.1007/ s11192-014-1229-3

Jhonnel Alarco, J.; Álvarez-Andrade, E. V.; Arroyo-Hernández, H. (2016). Diferencia de género en investigadores peruanos según Google Académico, Gaceta Sanitaria, 30(2), 160. https://doi.org/10.1016/j.gaceta.2015.12.003

Ke, Q. ; Ahn, Y. Y.; Sugimoto, C. R. (2017). A systematic identification and analysis of scientists on Twitter. PloS one, 12(4), e0175368. https://doi.org/10.1371/journal.pone. 0175368

Kim, Y. M. (2010). Gender role and the use of university library website resources: A social cognitive theory perspective. Journal of Information Science, 36(5), 603617. https://doi.org/10.1177/0165551510377709

Kjellberg, S.; Haider, J.; Sundin, O. (2016). Researchers' use of social network sites: A scoping review. Library \& Information Science Research, 38(3), 224-234. https://doi.org/10.1016/j.lisr.2016.08.008

Knobloch-Westerwick, S.; Glynn, C. J. (2013). The Matilda effect-Role congruity effects on scholarly communication: A citation analysis of Communication Research and Journal of Communication articles. Communication Research, 40(1), 3-26. https://doi. org/10.1177/0093650211418339

Larivière, V.; Ni, C.; Gingras, Y.; Cronin, B.; Sugimoto, C. R. (2013). Bibliometrics: Global gender disparities in science. Nature News, 504(7479), 211. https://doi. org/10.1038/504211a
Larivière, V.; Vignola-Gagné, E.; Villeneuve, C.; Gélinas, P.; Gingras, Y. (2011). Sex differences in research funding, productivity and impact: an analysis of Québec university professors. Scientometrics, 87(3), 483-498. https://doi.org/10.1007/s11192-011-0369-y

Leahey, E. (2006). Gender differences in productivity: Research specialization as a missing link. Gender \& Society, 20(6), 754-780. https://doi. org/10.1177/0891243206293030

Maliniak, D.; Powers, R. ; Walter, B. F. (2013). The gender citation gap in international relations. International Organization, 67(4), 889-922. https://doi.org/10.1017/ S0020818313000209

Martínez Lirola, M. (2010). Notas sobre la visibilidad y la invisibilidad de las mujeres en nuestra sociedad: el caso concreto de la Universidad de Alicante. Revista Nuevas Tendencias en Antropología, 2, pp. 37-58.

Martínez Lirola, M. (2011). Explorando la invisibilidad de mujeres de diferentes culturas en la sociedad y en los medios de comunicación. Palabra Clave, 13(1), pp. 161-173. https://doi.org/10.5294/pacla.2010.13.1.10

Mas-Bleda, A.; Aguillo, I.F. (2015). La web social como nuevo medio de comunicación y evaluación científica. Barcelona: Editorial UOC y EPI.

Mas-Bleda, A.; Thelwall, M.; Kousha, K.; Aguillo, I. F. (2014). Do highly cited researchers successfully use the social web? Scientometrics, 101(1), 337-356. https://doi.org/10.1007/s11192-014-1345-0

Mauleón, E.; Hillán, L.; Moreno, L.; Gómez, I.; Bordons, M. (2013). Assessing gender balance among journal authors and editorial board members. Scientometrics, 95(1), 87-114. https://doi.org/10.1007/s11192-0120824-4

Meishar-Tal, H.; Pieterse, E. (2017). Why do academics use academic social networking sites? The International Review of Research in Open and Distributed Learning, 18(1), https://doi.org/10.19173/irrodl. v18i1.2643

Messias, J.; Vikatos, P.; Benevenuto, F. (2017). White, man, and highly followed: Gender and race inequalities in Twitter. Proceedings of the International Conference on Web Intelligence Pages, WI 17, Leipzig, Germany - August 23 - 26, 266-274. https://doi. org/10.1145/3106426.3106472

Mikki, S.; Zygmuntowska, M.; Gjesdal, Ø. L. ; Al Ruwehy, H. A. (2015). Digital presence of Norwegian scholars on academic network sites-where and who are they? PloS one, 10(11), e0142709. https://doi.org/10.1371/ journal.pone.0142709

Mitchell, S. M.; Lange, S., Brus, H. (2013). Gendered citation patterns in international relations journals. International Studies Perspectives, 14(4), 485-492. https://doi.org/10.1111/insp. 12026

Nentwich, M.; König, R. (2014). Academia goes Facebook? The potential of social network sites in the scholarly realm. In: Bartling S., Friesike S. (eds) Opening 
Science. Cham: Springer, pp. 107-124. https://doi. org/10.1007/978-3-319-00026-8_7

Nicholas, D.; Rodríguez-Bravo, B.; Watkinson, A.; Boukacem-Zeghmouri, C.; Herman, E.; Xu, J.; Abriza, A.; Świgoń, M. (2017). Early career researchers and their publishing and authorship practices. Learned Publishing, 30(3), 205-217. https://doi.org/10.1002/leap.1102

Nilizadeh, S.; Groggel, A.; Lista, P.; Das, S.; Ahn, Y. Y.; Kapadia, A.; Rojas, F. (2016). Twitter's Glass Ceiling: The Effect of Perceived Gender on Online Visibility. In: Proceedings of the Tenth International AAAI Conference on Web and Social Media (ICWSM 2016), Cologne, Germany, May 17-20, pp. 289-298.

Orduña-Malea, E.; Martín-Martín, A.; Delgado-López-Cózar, E. (2016). La bibliometría que viene: ALMetrics (Author Level Metrics) y las múltiples caras del impacto de un autor. El profesional de la información, 25(3), 485-496. https://doi.org/10.3145/epi.2016.may.18

Orduña-Malea, E.; Martín-Martín, A.; Delgado-López-Cózar, E. (2016a). ResearchGate como fuente de evaluación científica: desvelando sus aplicaciones bibliométricas. El profesional de la información (EPI), 25(2), 303-310. https://doi.org/10.3145/epi.2016.mar.18

Orduña-Malea, E.; Martín-Martín, A.; Thelwall, M.; LópezCózar, E. D. (2017). Do ResearchGate Scores create ghost academic reputations? Scientometrics, 112(1), 443-460. https://doi.org/10.1007/s11192-017-2396-9

Ortega, J. L. (2015). Disciplinary differences in the use of academic social networking sites. Online Information Review, 39(4), 520-536. https://doi.org/10.1108/ OIR-03-2015-0093

Ortega, J. L. (2017). Toward a homogenization of academic social sites: A longitudinal study of profiles in Academia. edu, Google Scholar Citations and ResearchGate. Online Information Review, 41(6), 812-825. https://doi.org/10.1108/OIR-01-2016-0012

Paul-Hus, A.; Sugimoto, C. R.; Haustein, S.; Larivière, V. (2015). Is there a gender gap in social media metrics? In: Proceedings of ISSI 2015-15th International Conference of the International Society for Scientometrics and Informetrics, June 29-July 3, 2015, Isanbul, Turkey, pp 37-45.

Peñas, C. S.; Willett, P. (2006). Brief communication: Gender differences in publication and citation counts in librarianship and information science research. Journal of Information Science, 32(5), 480-485. https://doi. org/10.1177/0165551506066058

Potthoff, M.; Zimmermann, F. (2017). Is there a genderbased fragmentation of communication science? An investigation of the reasons for the apparent gender homophily in citations. Scientometrics, 112(2), 10471063. https://doi.org/10.1007/s11192-017-2392-0

Procter, R.; Williams, R.; Stewart, J.; Poschen, M.; Snee, H.; Voss, A.; Asgari-Targhi, M. (2010). Adoption and use of Web 2.0 in scholarly communications. Philosophical Transactions of the Royal Society of London A: Mathematical, Physical and Engineering Sciences, 368(1926), 4039-4056. https://doi.org/10.1098/ rsta.2010.0155

Rinaldi, A. (2014). Spinning the web of open science: Social networks for scientists and data sharing, together with open access, promise to change the way research is conducted and communicated. EMBO Reports, 15(4), 342-346. https://doi.org/10.1002/embr.201438659

Sugimoto, C. R.; Work, S.; Larivière, V.; Haustein, S. (2017). Scholarly use of social media and altmetrics: A review of the literature. Journal of the Association for Information Science and Technology, 68(9), 20372062. https://doi.org/10.1002/asi.23833

Thelwall, M.; Kousha, K. (2014). Academia.edu: social network or academic network?. Journal of the Association for Information Science and Technology, 65(4), 721-731. https://doi.org/10.1002/asi.23038

Tsou, A.; Bowman, T. D.; Sugimoto, T.; Lariviere, V.; Sugimoto, C. R. (2016). Self-presentation in scholarly profiles: Characteristics of images and perceptions of professionalism and attractiveness on academic social networking sites. First Monday, 21(4). https://doi. org/10.5210/fm.v21i4.6381

Van Rijnsoever, F. J.; Hessels, L. K. (2011). Factors associated with disciplinary and interdisciplinary research collaboration. Research Policy, 40(3), 463-472. https://doi.org/10.1016/j.respol.2010.11.001

Ward, J.; Bejarano, W. ; Dudás, A. (2015). Scholarly social media profiles and libraries: A review. Liber Quarterly, 24(4). https://doi.org/10.18352/lq.9958

Wildgaard, L.; Schneider, J. W.; Larsen, B. (2014). A review of the characteristics of 108 author-level bibliometric indicators. Scientometrics, 101(1), 125-158. https://doi.org/10.1007/s11192-014-1423-3

Woolley, R.; Sánchez-Barrioluengo, M.; Turpin, T.; Marceau, J. (2014). Research collaboration in the social sciences: What factors are associated with disciplinary and interdisciplinary collaboration?. Science and $\mathrm{Pu}-$ blic Policy, 42(4), 567-582. https://doi.org/10.1093/ scipol/scu074

Zhu, Y.; Purdam, K. (2017). Social media, science communication and the academic super user in the United Kingdom. First Monday, 22(11). https://doi. org/10.5210/fm.v22i11.7866 